\title{
Compositionally graded metals: A new frontier of additive manufacturing
}

\author{
Douglas C. Hofmann ${ }^{\text {a) }}$ \\ Engineering and Science Directorate, Jet Propulsion Laboratory, California Institute of Technology, Pasadena, CA \\ 91109, USA; and Keck Laboratory of Engineering Sciences, California Institute of Technology, Pasadena, CA \\ 91125, USA \\ Joanna Kolodziejska \\ Keck Laboratory of Engineering Sciences, California Institute of Technology, Pasadena, CA 91125, USA \\ Scott Roberts \\ Engineering and Science Directorate, Jet Propulsion Laboratory, California Institute of Technology, Pasadena, CA \\ 91109, USA; and Keck Laboratory of Engineering Sciences, California Institute of Technology, Pasadena, CA \\ 91125, USA \\ Richard Otis \\ Department of Materials Science and Engineering, Pennsylvania State University, University Park, PA 16802, USA \\ Robert Peter Dillon and Jong-Ook Suh \\ Engineering and Science Directorate, Jet Propulsion Laboratory, California Institute of Technology, Pasadena, CA \\ 91109, USA \\ Zi-Kui Liu \\ Department of Materials Science and Engineering, Pennsylvania State University, University Park, PA 16802, USA \\ John-Paul Borgonia \\ Engineering and Science Directorate, Jet Propulsion Laboratory, California Institute of Technology, Pasadena, CA \\ 91109, USA
}

(Received 8 April 2014; accepted 16 June 2014)

\begin{abstract}
The current work provides an overview of the state-of-the-art in polymer and metal additive manufacturing and provides a progress report on the science and technology behind gradient metal alloys produced through laser deposition. The research discusses a road map for creating gradient metals using additive manufacturing, demonstrates basic science results obtainable through the methodology, shows examples of prototype gradient hardware, and suggests that Compositionally Graded Metals is an emerging field of metallurgy research.
\end{abstract}

\section{INTRODUCTION}

Metal alloys are the most widely used structural materials, but they have limitations in their manufacturability and customization. For example, high strength alloys such as steel or titanium are generally machined from forged billets into hardware, assuring predictable mechanical properties. Their high melting temperatures generally make net-shaped casting difficult and their ability to have customized chemical composition or physical properties across a single part is limited by their consolidation from a homogeneous molten bath. The current work gives a progress report on the last four years worth of development in the science and technology behind forming net or near-net-shaped hardware with customizable gradient compositions utilizing additive manufacturing (AM). Using this

\footnotetext{
a)Address all correspondence to this author.

e-mail: dch@jpl.nasa.gov

DOI: $10.1557 /$ jmr.2014.208
}

technique, the mechanical and physical properties of a net-shaped part can be tuned through chemical composition, in addition to heat treating, surface engineering, or mechanical design. This allows for a new class of materials with dissimilar properties that would be difficult or impossible to obtain using other techniques - properties such as different densities, coefficients of thermal expansion, ferromagnetism, crystal structures and strengths, among many others. The design and fabrication of gradient alloys require a new approach compared to conventional metallurgy. Owing to the nearly infinite variable space provided by gradient metallurgy, a systematic roadmap for alloy design, AM techniques, and physical properties is required to make alloys with the desired functionality.

\section{AM WITH METAL}

AM processes (informally called three-dimensional printing) offer the ability to freeform hardware from a computer generated solid model, which is useful for developing a part without machining or fabricating a casting 
mold. ${ }^{1-3}$ Using AM with plastics has been well established over the last decade. Equipment is available at all scales, from tabletop printers to industrial machines capable of mocking up hardware for proof-of-concepts and producing actual consumer products. ${ }^{4}$ Figures 1(a)$1(\mathrm{~g})$ show polymer parts produced on two different classes of 3D printers. The 3D-Touch (produced by Bits from Bytes), shown in Fig. 1(a), is able to produce plastic parts from acrylonitrile butadiene styrene (ABS) and polylactic acid (PLA) and includes the ability to scale objects [Fig. 1(b)] and to use support material to facilitate overhanging features [Fig. 1(c)]. Larger machines, such as the Dimension printer (produced by Stratasys, Ltd., Rehovot, Israel), shown in Fig. 1(d), are able to print large components with relative complexity, which is used for mocking up entire spacecraft in miniature or life-size. Figure 1(e) shows a small-scale spacecraft while Fig. 1(f) shows a true-scale cube satellite. A full-sized replica wheel of NASA's Mars Rover Curiosity is shown in Fig. 1(g).

The benefits of freeform three-dimensional printing have also been extended to metal alloys as well as plastics. Some of the current technologies include direct metal laser sintering (DMLS), electronic beam melting, ultrasonic additive manufacturing (UAM), and laser engineered net shaping (LENS ${ }^{\circledR}$ also known as laser deposition). ${ }^{5-12}$ In contrast to the ease in which AM can be applied to polymers, where low melting temperatures and relatively high viscosities allow for precise and continuous deposition, AM with metals presents a number of difficulties. The high melting temperatures of metals limit the available heating methods and the viscosity of molten metal is much lower than thermoplastic polymers so continuous feeding is difficult. Figures 1(h) and 1(i) show two titanium parts that were built using DMLS. The part shown in Fig. 1(h)

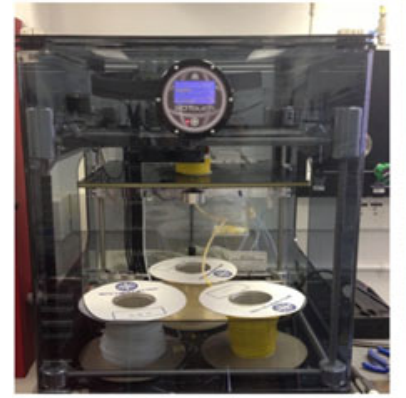

(a)

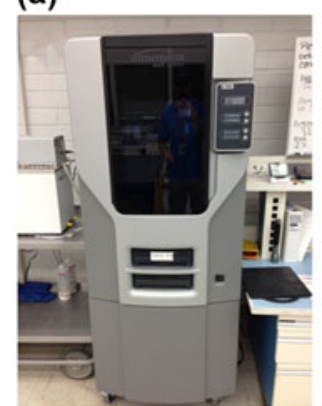

(d)

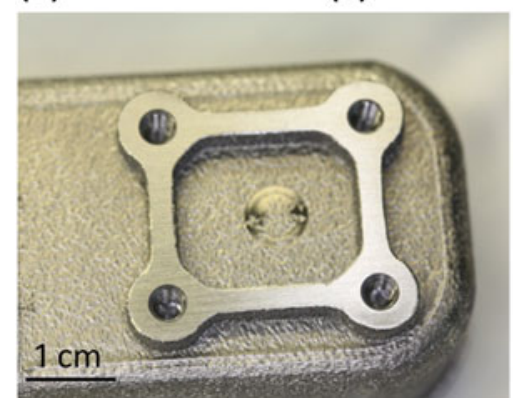

(h)

(b)

(e)
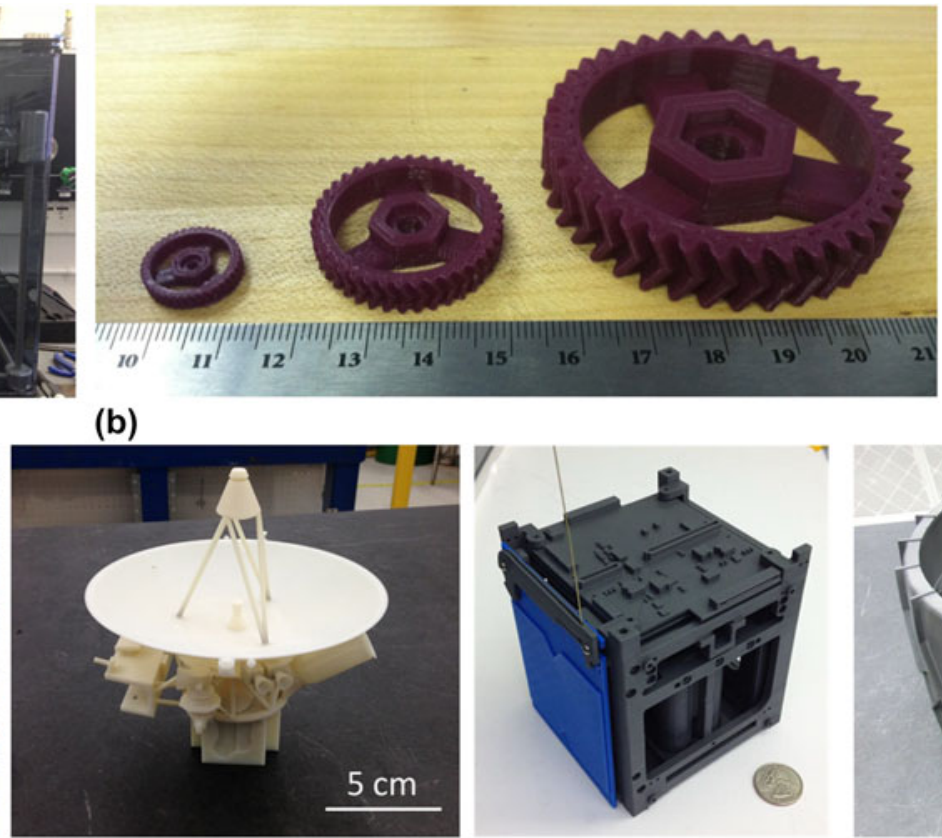

(f)

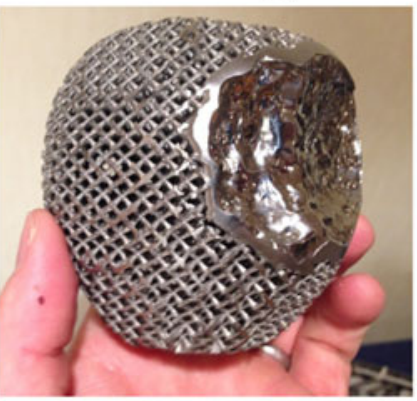

(i)

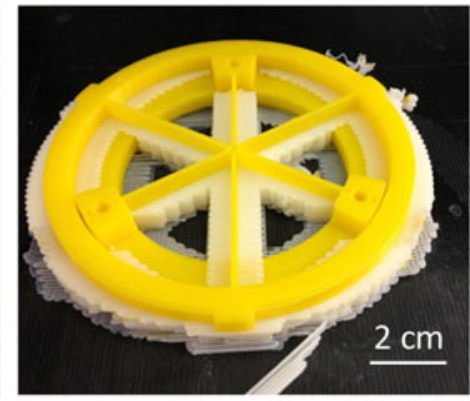

(c)

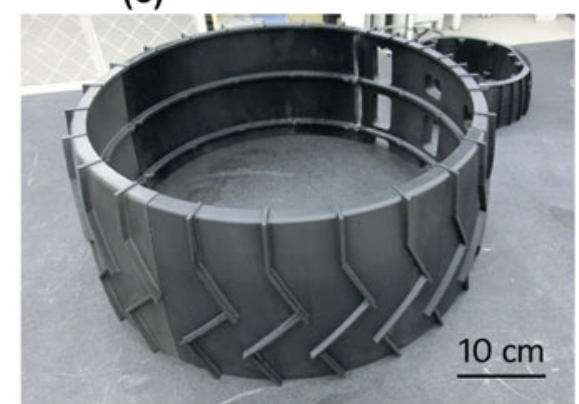

(g)

FIG. 1. (a-c) ABS plastic parts produced using the 3D Touch printer at NASA JPL. (d-g) An assortment of parts produced on the Dimension printer at NASA JPL, including (e) a small-scale spacecraft mock-up, (f) a full-size cube satellite, and (g) a replica of the Mars rover's wheel. (h) A Ti part produced using DMLS and (i) a cellular structure produced using DMLS. (j) Aluminum tape which has been consolidated on one end through UAM. 
has been finished-machined in one location, demonstrating the nearly fully dense structure. Fig. 1(i) is a cellular structure which illustrates the blind features that are accessible using the powder bed method of DMLS (produced by LayerWise, Leuven, Belgium). Figure 1(j) shows aluminum metal tape that has been consolidated using UAM (produced by Fabrisonic LLC, Columbus, OH). Among the most commercially mature technologies for metal printing are DMLS and laser deposition (LD), both of which use the consolidation of a powder using a laser heating source. DMLS uses a laser to raster over a bed of metal powder, consolidating only the powder that was subjected to the laser heating. After each layer has been consolidated, the workbench lowers and a new layer of powder is brushed over the workpiece and the rastering over the powder is repeated. Once the part is completed, it is removed from the loose powder and subsequently heattreated using hot isostatic pressing and then can be finish-machined to remove the rough outer surface layer. LD works by introducing the metal powder directly into the laser beam as the build head rasters back and forth over a workpiece. The laser creates a liquid melt pool as it rasters, allowing for a fully dense part to be built from the substrate up, layer-bylayer. Both DMLS and LD are being widely explored as a potential paradigm shift in the way that metal parts are fabricated. Versions of the machines now exist in industry, at universities, and in national laboratories, all with the aim of redefining rapid prototyping in metal.

Despite the promise of AM with metal, there are a number of significant drawbacks to its widespread utilization alongside established manufacturing. For AM to ultimately carve a space out of conventional manufacturing, there must exist a competitive case for using the technology (other than novelty). For an AM process to be selected over a conventional manufacturing technique, the drivers are

(i) The cost of the part fabricated through AM is lower than conventional machining.

(ii) The part is sufficiently complex that the AM process is faster than conventional machining or requires fewer man-hours to fabricate.

(iii) The part cannot be conventionally machined (e.g., the part has blind machining features).

(iv) The desired part is fabricated from a metal alloy which is difficult to machine so conventional machining would require longer fabrication times than AM.

(v) The resulting metal part can be standardized reliably so that each part does not need to be subjected to destructive quality-control testing.

(vi) The final part has a tailored composition that cannot be formed as a billet so conventional machining is not possible.

The three most significant drawbacks of metal AM are high infrastructure cost, long lead times, and lack of standards. These limitations stifle the use of AM for mass production parts or parts where precise mechanical properties are needed for performance. Large metal AM machines are expensive as is the powder feedstock, build times are extremely slow, AM parts require finish machining, and unless the final part is complex, machining from a billet is faster. In some cases, however, AM is a viable technology for both production parts and prototyping. For example, complex components (such as cellular structures or other geometries with low relative density) made from titanium are excellent candidates for DMLS. Titanium is hard and difficult to machine, especially in a complex part where only a small percentage of the original billet remains. In these parts, DMLS can satisfy the first five drivers listed above, and therefore has a niche application that is more competitive than conventional machining. Figure 2 demonstrates such a case where an AM application could be applied to hardware. Optics mounts, which are metal mounts that hold mirrors, are typically complex and require precision flexures to grasp and tilt mirrors. The mirror mount shown in Figs. 2(b) and 2(c) was fabricated out of a large billet of Ti into the final part (image courtesy of Paul Gardner, NASA JPL). The original dimensions of the billet exceeded the height of the small flexures, which demonstrates that far more than $50 \%$ of the Ti was removed during fabrication. The schematic in Fig. 2(a) shows that for a part like this, it may be cost effective to build the flexure in an additive process by depositing only the material needed for the flexure onto the Ti substrate. Figure 2(d) shows a Ti-6Al-4V (Ti-6-4) block where a post of Ti-6-4 was deposited using LD (produced by RPM Innovations, South Dakota). The Ti-block was then conventionally machined into the mirror flexure, as shown in Figs. 2(e) and 2(f), using the same conventional machining that was used to fabricate the hardware, as shown in Fig. 2(b). This demonstrates that the AM process is effective when it is applied strategically, as with a part that would require more than $50 \%$ removal of a billet to fabricate.

\section{COMPOSITIONALLY GRADED METALS USING AM}

Early in the development of LD techniques, it became apparent that the process could be utilized to fabricate functionally or compositionally graded metals (i.e., gradient alloys) by mixing elemental or alloy powders as they were introduced into the laser. ${ }^{13,14}$ Because the LD process creates a melt pool in the wake of the laser, the technique can be used to perform layer-by-layer alloying, where each deposited layer can have a different composition than the previous layer through the blending of different feedstock powders. This technique fundamentally changes the paradigm for the fabrication of metal hardware because 


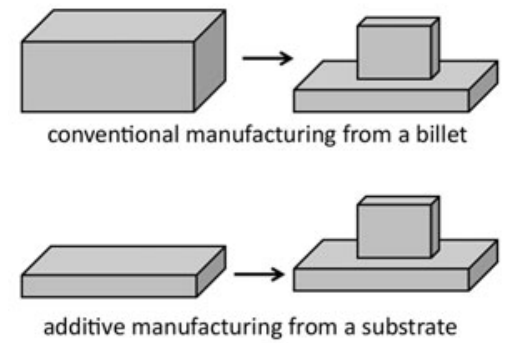

(a)

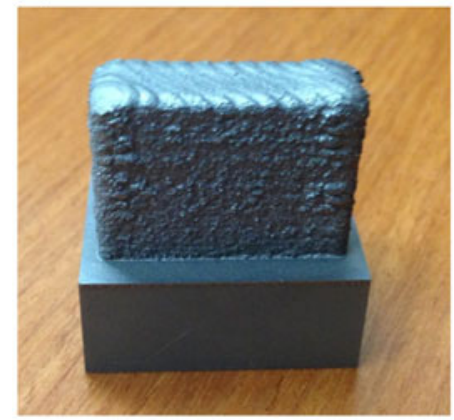

(d)

(e)

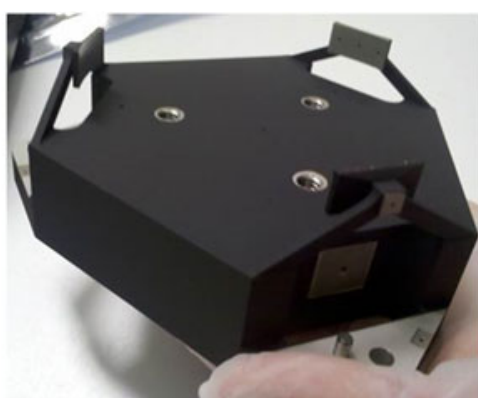

(b)

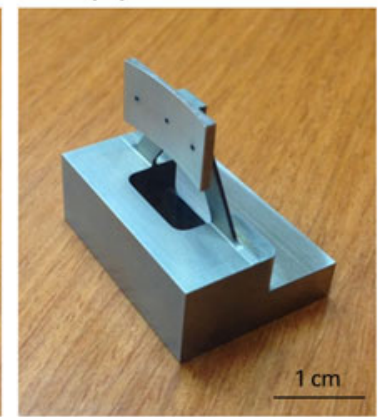

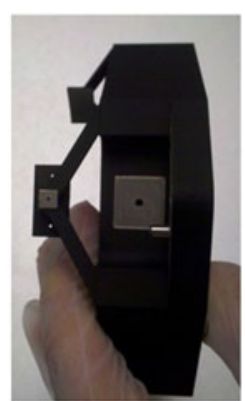

(c)

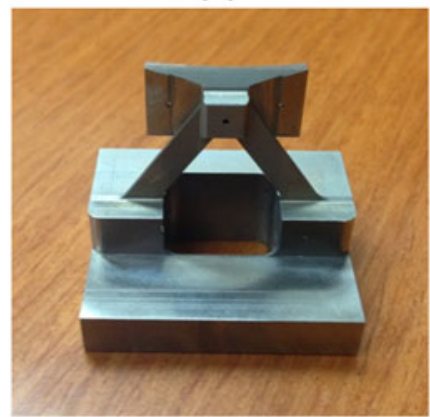

(f)

FIG. 2. (a) Schematic of how two identical parts can be achieved through conventional machining (removal of material) and AM (addition of material). (b, c) A titanium mirror mount with small flexures used to hold a mirror. The part was machined from a billet. (d) A block of Ti that was deposited onto a Ti substrate using LD at RPM Innovations. (e, f) The same flexure as in (b, c) machined from the part in (d), demonstrating that the additive process requires far less machining than conventional techniques.

it allows, for the first time, chemical composition to be used as a design parameter. Traditionally, surface engineering (e.g., cladding, plating, coating, and shot peening) and localized heat treating are two ways in which the mechanical or physical properties of the metal alloy can be tailored in specific regions. This can be done for wear, corrosion resistance, or for hardening in a localized region, among others. In contrast, LD allows for freeform hardware to be fabricated from computer models so that the composition of the internal structure of the part can be altered. This also allows metal parts to be fabricated that sample large regions of compositional space, which is extremely useful for basic metallurgy and alloy development studies.

Essentially, any AM technique can be used to fabricate a gradient alloy. By definition, AM means that a part is fabricated one layer at a time in an "additive" process. Because of this, the composition of the material being deposited onto the previous layer can always be different, regardless of whether the feedstock is polymer, metal powder, or metal wire. ${ }^{13-16}$ However, some AM techniques are far more conducive to changes in feedstock composition, particularly those where the feedstock is introduced into the heating source at the build head, and not in workpiece. Although gradient alloys have largely only been demonstrated in polymers and in metals using LD, it is possible to consider other techniques as well. Electron beam freeform fabrication (EBF3), developed at NASA Langley, ${ }^{17-19}$ works by introducing metal wire into an electron beam. Twin arc spraying and thermal spray coating use metal wire or powder as a feedstock for spray deposition. In both of these techniques, two or more wires or powders could be introduced into the building head (or spray gun) to fabricate gradient alloys. It is more challenging to fabricate gradient alloys using AM techniques that require a powder bed, as with DMLS. To change composition, the powder must be drained from the system, modified, and then reintroduced over the workpiece to create the gradient. In contrast, LD is an optimal technique for creating gradient alloys because powder metal feedstock is sprayed into the melt pool created by the laser. Today's commercial LD systems allow for up to four different feedstock nozzles to deliver powder to the laser, leading to a nearly infinite combination of potential gradients to be formed. Figure 3(a) shows several compositionally graded alloys being produced using LD at RPM Innovations. A schematic of the build head is shown in Fig. 3(b) with 4 powder feeders introduced into the YAG laser. In the LD process, the laser melts each layer, which alloys the powder and forms a metallurgical bond with the previous layer. Build times are slow and the parts exhibit large amounts of residual heat, resulting in annealed microstructures after cooling. Depending on the desired mechanical or physical properties in the final part, the gradient alloy can be designed to have a smooth graded transition from one metal to another 
or it can proceed in large compositional steps. A smooth grading might be desirable for reducing internal stresses (as with a part subjected to large temperature gradients) while a stepped gradient might be useful for sharp transitions in properties (like magnetism, for example). In any case, the additive building process allows for a greater control over the properties of metal parts than any previous metallurgy technique.

Figures 3(c)-3(h) show schematics of the types of compositionally graded alloys that can be obtained using the LD process, where the colors represent alloys of different compositions and blended colors represent that a metallurgical transition has occurred from one to another. Figure 3(c) illustrates a linear gradient where a part is changed to a completely different alloy in a rule-of-mixtures gradient. The step size or compositional accuracy of each layer is largely based on the complexity of the LD machine being used. Figure 3(d) shows an alloy with a step-transition in composition, where a pure alloy (or element) is deposited for a prescribed distance and in the transition from one layer to the next, the composition is changed completely to another alloy. These gradients only work if the transition layer is free of brittle phases (discussed in Sec. IV). Figure 3(e) illustrates multiple gradients where one material has been strategically added throughout a part. This could be done to take advantage of thermal expansion or magnetism differences between the two deposited layers. Figure 3(f) shows a transition through three distinct material compositions, first from blue to green, then from green to orange. These gradients are useful when two compositions cannot be graded without the formation of brittle phases and yet they can both be graded to a third intermediate material. Later, it is shown that this technique is useful for gradients from $\mathrm{Fe}$ and $\mathrm{Ti}$, which do not mix well but can both be graded to refractory metals, such as $\mathrm{Nb}, \mathrm{V}$, and Ta. Figures 3(g) and 3(h) show two classes of metalmatrix-composites (MMCs) that can be fabricated with LD. The alloy in Fig. 3(g) shows an insoluble material that has been graded with a matrix. This works when one of the powders being introduced into the laser has a much higher melting temperature than the other so that the particles do not alloy and form inclusions. In the present study, we show that gradients from $\mathrm{Ti}-\mathrm{TiC}$ are possible using this technique. Lastly, MMCs can also be formed utilizing chemical desegregation, a technique which has been widely exploited in the bulk metallic glass research area by adding $\mathrm{Be}$ and $\mathrm{Cu}$ to $\mathrm{Zr}-\mathrm{Ti}$-Nb alloys to form two-phase MMCs. ${ }^{19,20}$

\section{USING PHASE DIAGRAMS AS MAPS}

The tremendous advantage of using gradient AM techniques is the ability to fabricate custom compositions, using binary, ternary, or quaternary phase diagrams as a map between desired compositions. In contrast to

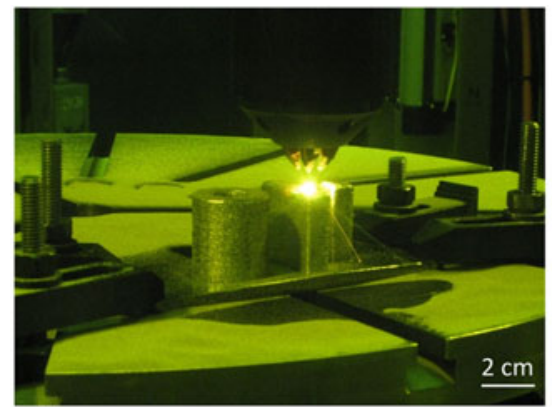

(a)

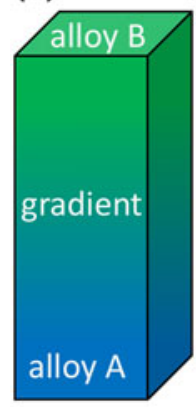

(c)

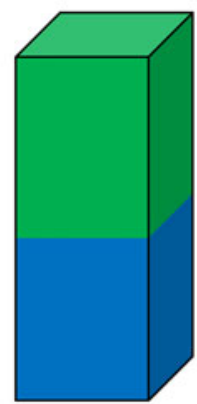

(d)

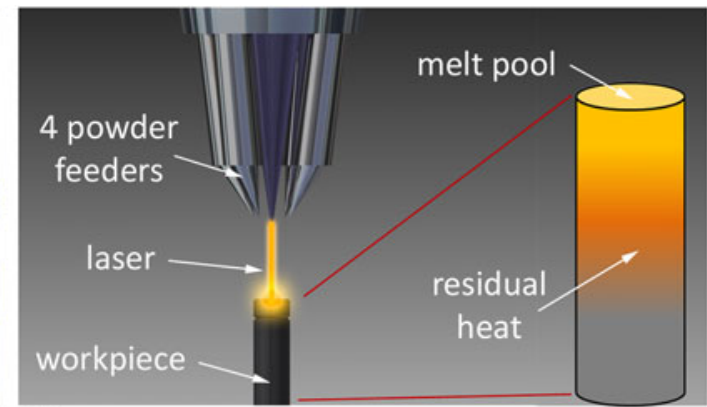

(b)

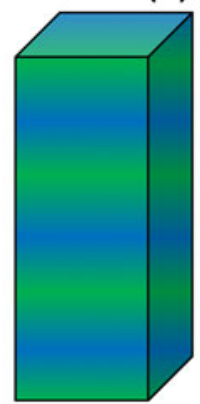

(e)

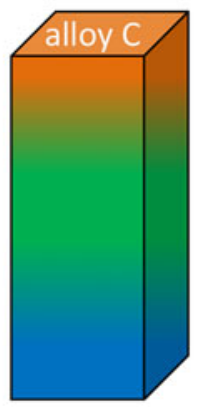

(f)

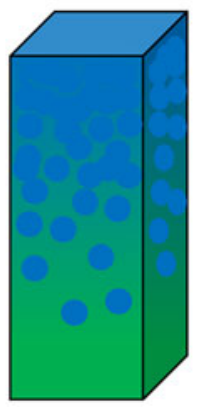

(g)

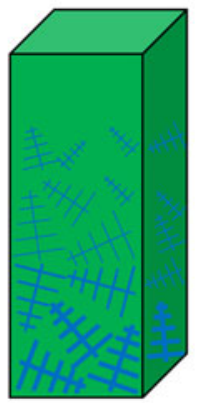

(h)

FIG. 3. (a) Gradient alloys being produced at RPM Innovations. (b) A schematic of the build head during the LD process. (c-h) Schematics of some of the different compositionally graded alloys that are possible using the LD process. These include the formation of gradients from one alloy to another and the formation of metal matrix composites $(\mathrm{g}, \mathrm{h})$. 
traditional alloy development, where multicomponent alloys are made one point at a time until a region of a phase diagram is understood, the gradient process allows for many compositions along a line to be fabricated in the same part. This is desirable for alloy development, because nanomechanics can be used to characterize many compositions in a single sample without having to make them individually. It is also desirable for transitioning from one alloy to another to exploit the mechanical or physical properties at each end of the gradient. For each gradient alloy, there must be a defined "gradient path" selected for the AM building process. There may be many of these paths that could be selected depending on the functionality required by part. The path could be linear, representing the most direct route from one composition to another; the path could be curved to avoid unwanted phases; or the path could be discontinuous to create a step in compositions. Examples of these types of paths are shown in a schematic ternary phase diagram in Fig. 4(a). The most direct route from one alloy to another is a simple mixture of one powder to another linearly (shown with a red line).
However, if this route encounters an unwanted phase (shown schematically in the colored regions), then a path could be designed to circumvent that region. With computer control of the gradient path, complex routes are possible. In some cases, binary transitions are desirable for reducing complexity. In these cases, alloys with minority alloy elements can be graded to a pure metal and then blended with another pure metal. With information about different crystal phases that form in multicomponent systems, computer programs can be used to design gradient paths that optimize the desired function of the graded part, whether it is a high fracture toughness transition from one material to another, a gradient of mechanical or physical properties (such as melting temperature, thermal expansion, or magnetism, for example), or stepped transitions from one alloy to another to avoid unwanted phases.

Developing gradient paths for compositionally graded alloys is greatly assisted through the use of predictive phase diagram modeling. In the current research, the authors developed predictive gradient paths

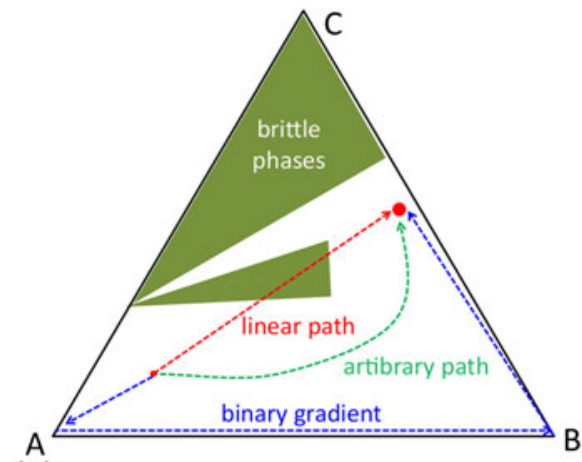

(a)

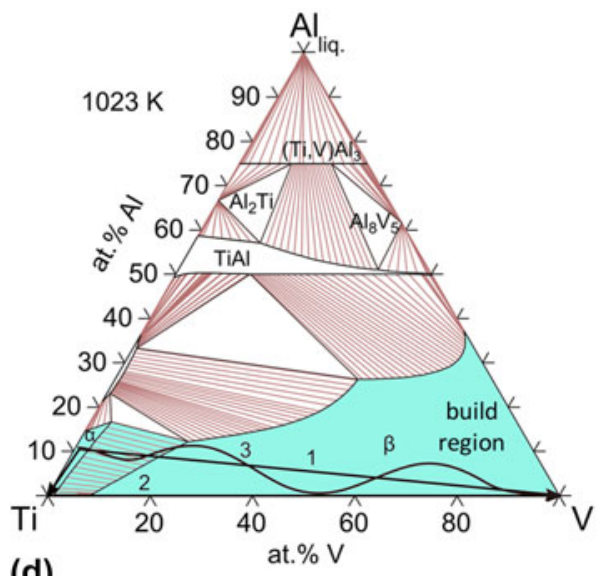

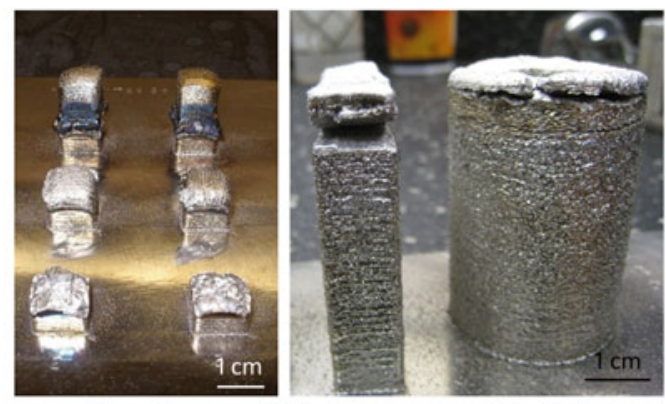

gradient alloys cracked from formation of brittle phases

(b)

(c)

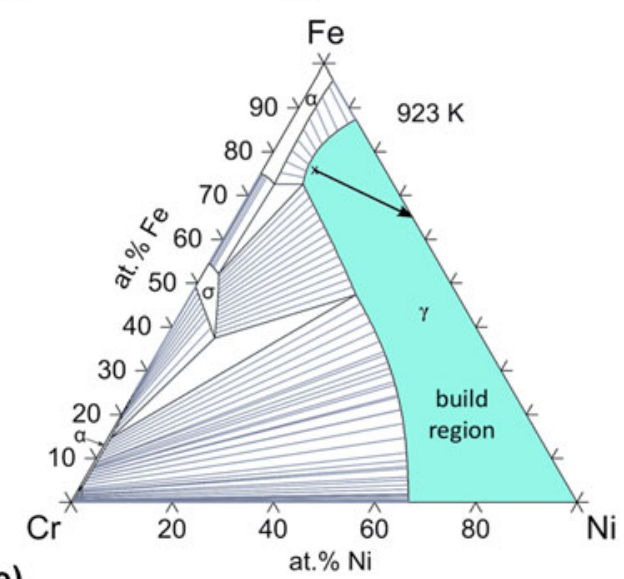

(e)

FIG. 4. (a) A schematic of a ternary phase diagram showing possible gradient paths from one alloy to another across a three-element phase space. Several routes are possible, based on factors such as the avoidance of brittle phases. (b, c) Gradient alloys which cracked during fabrication. (b) A failed gradient from Ti to Invar and (c) a failed gradient from Ti to 304L stainless steel. (d) A calculated Al-Ti-V phase diagram showing a gradient from $\mathrm{Ti}-6 \mathrm{Al}-4 \mathrm{~V}$ to pure $\mathrm{V}$ showing three different gradient paths: (1) a linear gradient, (2) removing $\mathrm{Al}$ and then performing a binary Ti-V gradient, (3) an arbitrary path. The blue region is calculated to be free of brittle intermetallic phases and is comprised of hcp and bcc phases. (e) A calculated phase diagram for the $\mathrm{Fe}-\mathrm{Ni}-\mathrm{Cr}$ system showing a gradient from 304L stainless steel to Invar 36. In this diagram, the blue region has been calculated to be all single phase austenite, which simplifies the build due to the absence of brittle phases. 
for the LD process using the CALPHAD method. ${ }^{21-24}$ Using information about the desired composition and processing conditions of the samples, phase diagrams can be calculated which predict the phases that will be present in the solidified gradient alloy. Figure 4(d) shows such a phase diagram for the Ti-Al-V system, where three different gradient paths are shown for the transition from Ti-6-4 to pure elemental V. Although details about this gradient will be published elsewhere, the three paths indicate the different ways that Ti-6-4 can be transitioned to $\mathrm{V}$ without encountering brittle intermetallic phases. These include the linear route (which was developed experimentally and is labeled 1), the route where Ti-6-4 is transitioned to pure $\mathrm{Ti}$ and then pure $\mathrm{V}$ (labeled route 2), and the arbitrary path (labeled route 3 ). A similar gradient from Ti-6-4 to pure $\mathrm{Nb}$ (another refractory metal) is discussed later, as shown in Fig. 7. Figure 4(d) shows a calculated phase diagram for the transition from 304L stainless steel $\left(\sim \mathrm{Fe}_{68} \mathrm{Cr}_{20} \mathrm{Ni}_{10} \mathrm{Mn}_{<1} \mathrm{Si}_{<1}\right.$ in wt\%) to Invar $36\left(\mathrm{Fe}_{64} \mathrm{Ni}_{36}\right.$ in $\left.\mathrm{wt} \%\right)$. The calculated phase diagram accurately predicts the single phase austenite at all compositions of the gradient, which was verified experimentally. In many alloy systems, however, obvious transitions between alloys of interest do not exist. For example, Fig. 4(d) shows that a transition from Ti-6-4 to pure V is free of brittle phases; however, a transition from Ti-6-4 to pure $\mathrm{Al}$ would form many brittle phases. To test the effect of these transitions, several gradients were deliberately steered toward locations in composition space where brittle compositions were expected. Figures 4(b) and 4(c) show failed gradients in a transition from Ti to Invar 36 and another one from $\mathrm{Ti}$ to $304 \mathrm{~L}$ steel. As expected, the thermal stresses associated with the AM building process cause cracking in the brittle ordered phases that form. In some cases, the gradient alloy breaks off and the deposition is halted, while in other cases a crack is visible at a location along the gradient. Cross-sectional scanning electron microscopy (SEM) image on these alloys verifies that brittle phases have formed and cracked (see Fig. 7 for more detail).

\section{A ROADMAP FOR CREATING GRADIENT ALLOYS}

Compositionally graded metals offer both the opportunity to explore basic science research as well as to design hardware with properties that cannot be attained using traditional manufacturing. Designing a gradient alloy for a particular application starts by identifying a multifunctional part that requires the gradient. The need may arise from an application with problematic mechanical property issues (e.g., a thermal expansion mismatch), a metal part that needs to be joined to a dissimilar metal part (e.g., a titanium to steel weld), or a desire to make the design and function of a part more elegant (e.g., a single-piece component with multifunctional mechanical properties). Figure 5 shows a finite element model of a typical part that might benefit from a gradient alloy; an automobile valve stem. The model shows that at $1000 \mathrm{~K}$ the joint with the gradient alloy has an approximately ten times lower stress due to the lack of thermal expansion. The gradient joint has an order of magnitude lower stress at $1000 \mathrm{~K}$ due to thermal expansion.

The next step in gradient design is to identify the alloys that, when combined, could achieve the desired properties required by the application. For example, if one side of a metal part is exposed to high temperatures and the other side needs to be low density, several gradient choices are possible. The high temperature side could be a refractory metal, such as $\mathrm{Ta}, \mathrm{Nb}, \mathrm{V}, \mathrm{Mo}$, or $\mathrm{W}$ while the low density metal could be $\mathrm{Al}$ or $\mathrm{Ti}$. Once some potential alloys are selected, the gradient needs to be designed in such a way as to satisfy the desired properties of the final part. Advanced computer modeling can be used to optimize the design of a part to exploit differences in mechanical properties between alloys. Once the part is modeled, a gradient path must be developed to transition between the alloys of interest without creating brittle or unwanted phases. If this is demonstrated successfully, then an AM building technique must be selected to fabricate the part. Although LD was used in the current work, this may not be the best solution for all desired gradients. For example, some metal alloys may be difficult to obtain in powder form but are readily available in wire. If the gradient alloy

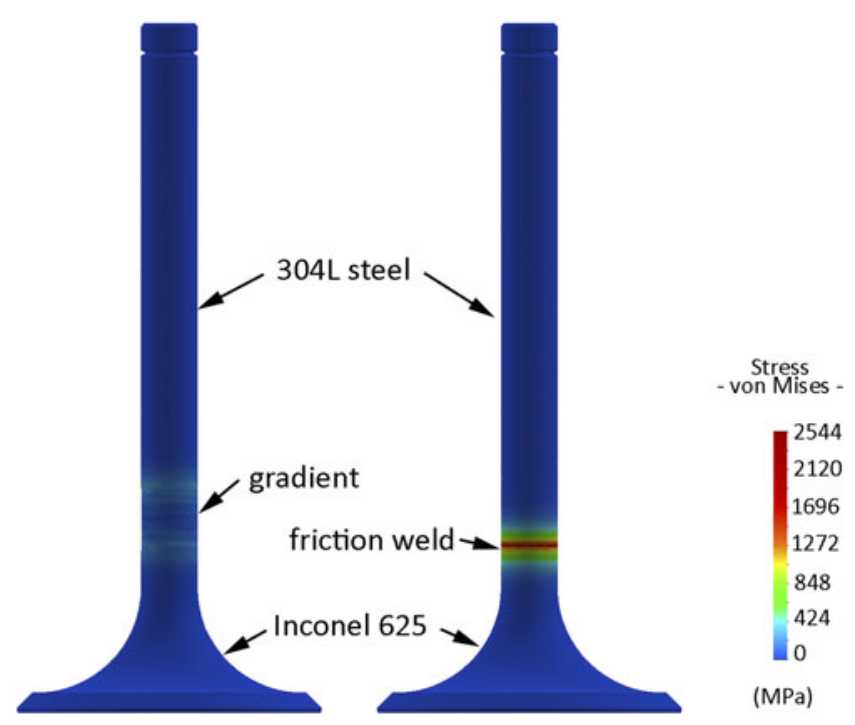

FIG. 5. A finite element model showing elastic mismatch in two dissimilar metal automobile valve stems at $1000 \mathrm{~K}$. The figure on the left shows a valve with a 304L stainless steel stem connected to an Inconel 625 valve via a $2.5 \mathrm{~cm}$ long gradient of composition. On the right, the gradient is replaced with a friction weld. The stress at the joint of the friction welded part has an approximately ten times higher stress than the compositionally graded alloy. 
has a small thickness, but a large surface area, then spray coating the gradient might be a viable option. Spray coating can be used to fabricate gradient alloys by changing the composition of the powder or wire that enters the spray nozzle.

\section{COMPOSITIONALLY GRADED METALS AS A NEW BASIC SCIENCE RESEARCH AREA}

The current work demonstrates that fabricating parts using compositionally graded metals is only a small part of the potential for the technology. In the process of characterizing the gradient alloys that were fabricated, numerous areas of fundamental science were explored. As demonstrated previously, compositionally graded alloys can be developed to rapidly explore large sections of phase diagrams experimentally. The resulting phases that form can also be predicted using phase diagram calculations and other software, adding a new dimension to alloy development and metallurgy. The process is also conducive for studying the change in mechanical and physical properties across a metallurgically bonded part. This could include alloys that exhibit dramatic differences in density, crystal structure, hardness, toughness, thermal expansion, melting temperature, among many others. Figure 6 illustrates some of the differences in properties that can be obtained with gradient metallurgy. Figure 6(a) is a compiled 3D x-ray scan that shows the transition from hexagonally closed packed (hcp) Ti-6-4 to body centered cubic (bcc) V. The plot was constructed by slicing a compositionally graded alloy into $1 \mathrm{~mm}$ thick slices and then performing x-ray diffraction (XRD) on both sides of each slice and then mapping the results in three dimensions. The plot shows that the hcp Ti alloy transitions into a bcc alloy as the percentage of $\mathrm{V}$ in the alloy is increased. Once the alloy transitions to bcc, the peaks in the scan shift to the right (indicating an increasing lattice size) until the scan arrives at the peaks from pure $\mathrm{V}$. Individual scans from the pure Ti-6-4 side, the pure $\mathrm{V}$ side and one intermediate composition are shown in Fig. 6(b). A Ti-V compositionally graded alloy therefore not only exhibits dramatic differences in mechanical/physical properties (hardness, density, melting temperature, etc.), but it also transitions across crystal structures. This result is of fundamental interest to researchers who study diffraction and crystal structure.

Figure 6(c) shows a plot of electron dispersive x-ray spectrometry (EDS) measurements of composition across a 316L stainless steel $\left(\sim \mathrm{Fe}_{71} \mathrm{Cr}_{16} \mathrm{Ni}_{10} \mathrm{Mo}_{2} \mathrm{Mn}_{1}\right.$ in wt $\left.\%\right)$ to Invar 36 gradient alloy. The alloy was fabricated by depositing $\sim 5 \mathrm{~mm}$ of pure $316 \mathrm{~L}$, linearly mixing Invar 36 for approximately $15 \mathrm{~mm}$ and then depositing $\sim 5 \mathrm{~mm}$ of pure Invar 36. Details about step-size, laser power, and compositional analysis will be shown elsewhere; however, what is of note is the compositional similarities between the two alloys despite their differences in key mechanical
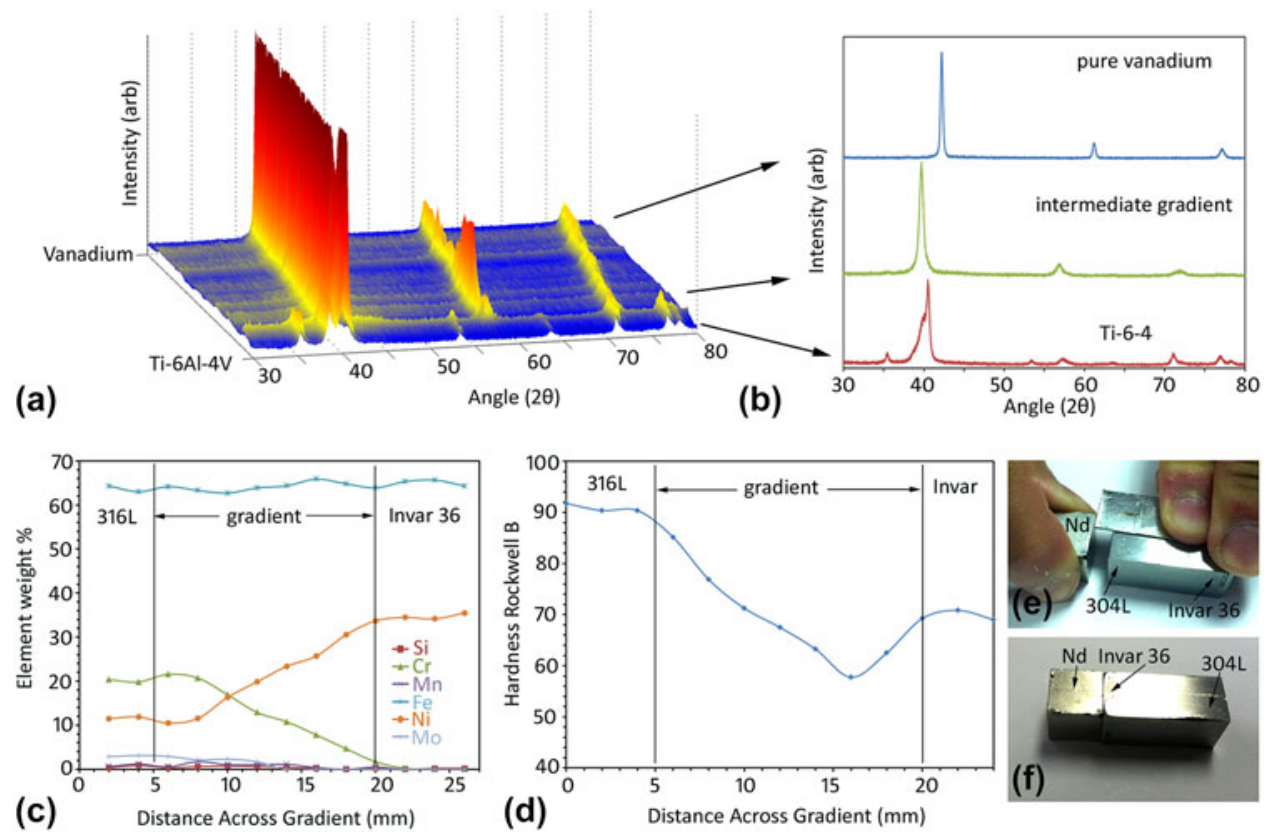

FIG. 6. Examples of basic science research that the gradient alloy process allows. (a) A 3D XRD scan from a Ti-6Al-4V to V gradient alloy taken from $1 \mathrm{~mm}$ slices of the alloy. (b) Selected XRD scans from the compiled figure in (a). (c) Chemical composition data from a $316 \mathrm{~L}$ to Invar 36 gradient alloy showing the intermediate compositions between the alloys. (d) Rockwell hardness across the gradient alloy in (c) showing all softaustenite is present in the gradient region. (e, f) Experiment showing the difference in ferromagnetism between the two sides of a $304 \mathrm{~L}$ to Invar 36 gradient alloy. (e) The $304 \mathrm{~L}$ side of the gradient is only weakly magnetic when a $\mathrm{Nd}$ rare earth magnet is brought close. (f) The Nd magnet is strongly attracted to the Invar side of the gradient alloy. 
properties, such as thermal expansion, magnetism, and hardness. Both alloys have similar percentages of $\mathrm{Fe}$, but Invar 36 has an increased percentage of $\mathrm{Ni}$ and no $\mathrm{Cr}$, while 316L has both $\mathrm{Ni}$ and $\mathrm{Cr}$. At each intermediate composition in the alloy, a single phase of soft austenite was formed, as verified by hardness measurements [Fig. 6(d)], XRD analysis, and phase diagram calculations [Fig. 4(e)]. Despite the subtle changes in composition between the two ends of the gradient alloy, several key mechanical properties were graded significantly. Figures 6(e) and 6(f) show experimentally the differences in ferromagnetism between the two sides of the gradient. A powerful rare-earth magnet does not attract the $316 \mathrm{~L}$ side of the gradient but does attract the Invar 36 side. Similarly, the coefficient of thermal expansion (CTE) in both sides of the gradient is significantly different, considering Invar 36 has a nearly zero CTE at low temperatures. These measurements will also be included in another study.

Figure 6 demonstrates that compositionally graded alloys allow for a broad range of basic science studies, largely based on the fact that these alloys cannot be fabricated utilizing other processing techniques. Among these, information about crystal structures, intermediate alloy compositions, physical properties, and mechanical properties are the most interesting because these features of an alloy cannot be changed easily using mature metallurgy techniques. Gradient alloys change the paradigm for how properties can be distributed in a material, making them of fundamental interest.

\section{HARDWARE WHICH EXPLOITS GRADIENT COMPOSITIONS}

To demonstrate potential applications of compositionally graded metal alloys, several prototype parts were fabricated using alloy combinations that were successfully developed during experimentation. Figures 7(a)-7(e) show a prototype gradient rocket-nozzle that was developed using a gradient from Ti-6-4 to pure $\mathrm{Nb}$. The schematic in Fig. 7(a) shows the full design of a one-piece rocket where the body is made of low-density Ti-6-4 but the nozzle, where the majority of the heat load is experienced, is a high-melting temperature refractory metal $(\mathrm{Nb})$. In the prototype, only the nozzle section was fabricated, shown as a model in Figs. 7(b) and 7(c) and after the LD process, as shown in Fig. 7(d). The whole nozzle section of the part is $\mathrm{Nb}$ and after the neck, the part transitions via linearly to Ti-6-4. The final part after surface machining is shown in Fig. 7(e). This work is of interest to NASA, as NASA's Marshall Space Flight Center (MSFC) has already been testing a monolithic Ti 3D-printed rocket nozzle. $^{25}$

Figures 7(f)-7(j) show another high-temperature application for compositionally graded alloys: An auto- motive valve stem. In high-performance automobiles, valves are fabricated from a high-temperature Ni-alloy, Inconel $625\left(\mathrm{Ni}_{63} \mathrm{Cr}_{20} \mathrm{Mo}_{8} \mathrm{Fe}_{5} \mathrm{Nb}_{4}\right.$ in wt $\left.\%\right)$, and are then friction-welded to a stainless steel stem. The friction weld is often prone to failure in these parts, due to abrupt transition in the weld. Figure $7(\mathrm{~g})$ shows three netshaped gradient valves being 3D printed using LD and the parts after fabrication but before finish machining. Figures 7(h) and 7(i) show two of the valves after finish machining where the gradient in composition is virtually undetectable in the fully dense part. The gradient from $304 \mathrm{~L}$ to Inconel 625 occurs over $2.5 \mathrm{~cm}$ in the neck but the entire part is a single phase of austenite.

Figures 7(k)-7(o) show parts fabricated using a gradient alloy that transitions from 304L stainless steel to Invar 36. A model of a mirror with a triangular isogrid backing is shown in Fig. 7(k) where the part has been designed so that the isogrids are made from steel which transitions to low CTE Invar at the mirror. The part was built up by depositing Invar on a 304L plate and the composition was graded back to $304 \mathrm{~L}$ at the completion of the build, shown in Fig. 7(1). The part was finished-machined to remove the surface roughness of the part (which was overbuilt for practical reasons) and the final mirror is shown in Figs. 7 (m) and 7(n). An experimental test specimen of a gradient from 304L to Invar is shown in Fig. 7(o) before surface machining. The gradient is detectable due to the lack of oxide on the Ni-rich Invar side of the gradient. After surface machining, a 304L plate was laser-welded across the entire gradient to demonstrate that every composition throughout the alloy can still be conventionally welded, shown in Fig. 7(p).

A gradient alloy which transitions from Ti-6-4 to 304L stainless steel is shown in Figs. 7(q)-7(t). Due to the many intermetallic phases present in the $\mathrm{Ti}-\mathrm{Fe}$ phase diagram, a direct compositional gradient from Ti-6-4 to 304L (or Invar) was not possible (see the failed gradients in Fig. 4). Instead, both Ti-6-4 and stainless steel can be compositionally graded to refractory metals (in this case V). A gradient from Ti-6-4 to V to 420 stainless steel is shown in Fig. 7(q). A slightly different gradient from Ti-6-4 to $\mathrm{V}$ to 304L is shown in Fig. 7(r) with a visible crack in the gradient. The crack is evident when the sample is polished [see Fig. 7(s)]. The crack was examined via SEM and the crack was only visible in a light colored phase shown in Fig. 7(t). EDS on that composition showed that the alloy cracked at $\mathrm{Fe}_{60} \mathrm{~V}_{30} \mathrm{Cr}_{10}$ and only at this composition.

Finally, Figs. 7(u) and 7(v) show a Ti-TiC MMC which has been fabricated to illustrate the process of forming a composite by utilizing an insoluble powder. At the laser power $(\sim 800 \mathrm{~W})$ used to melt Ti powder, there is insufficient heat to melt the TiC powder, which forms an inclusion in a Ti matrix. Just like with compositionally graded alloys, the volume fraction of the TiC can 


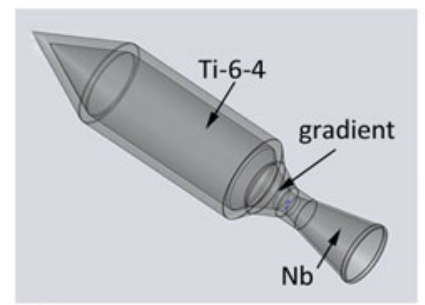

(a)

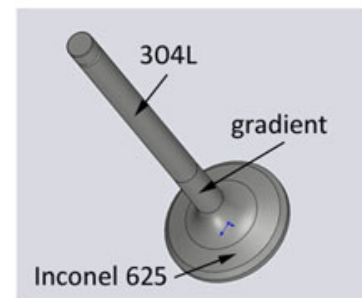

(f)

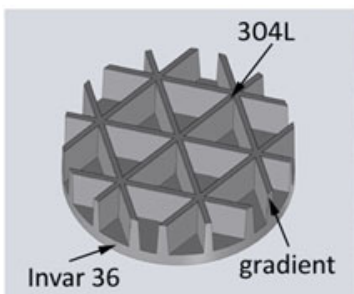

(k)

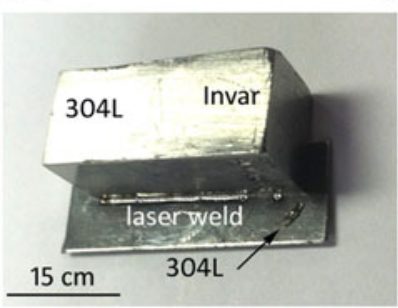

(p)

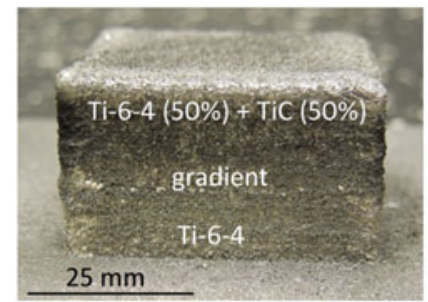

(u)

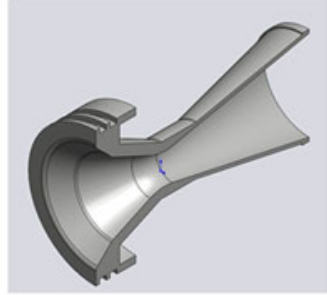

(b)

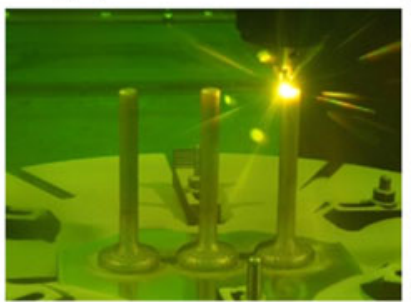

(g)

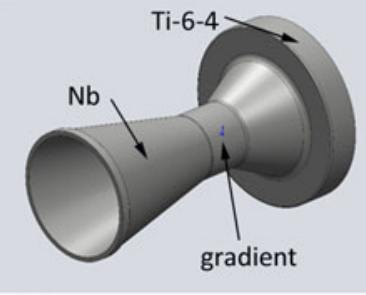

(c)

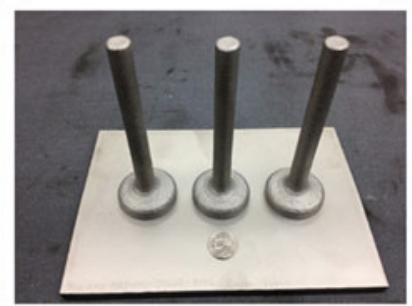

(h)

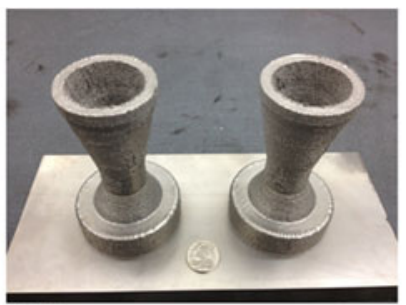

(d)

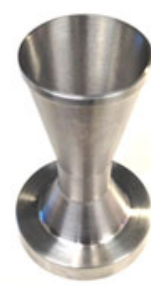

(e)

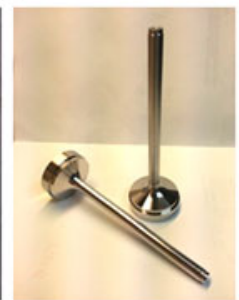

(i)

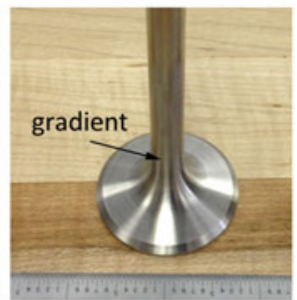

(j)

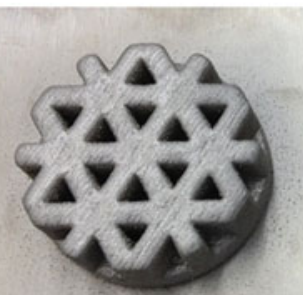

(I)

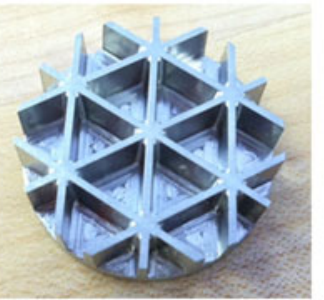

(m)

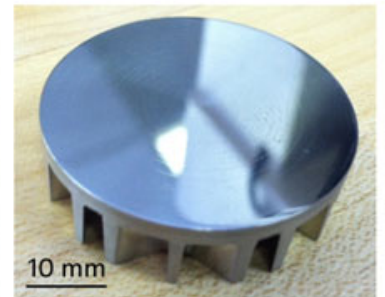

(n)

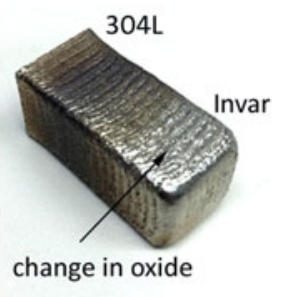

(o)

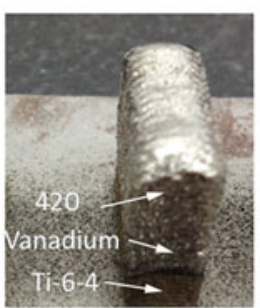

(q)

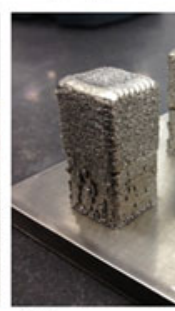

(r)

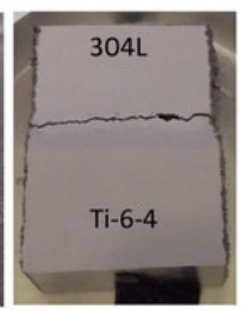

(s)

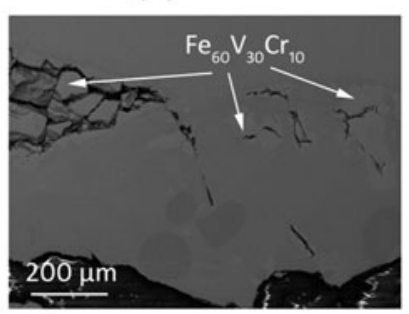

(t)

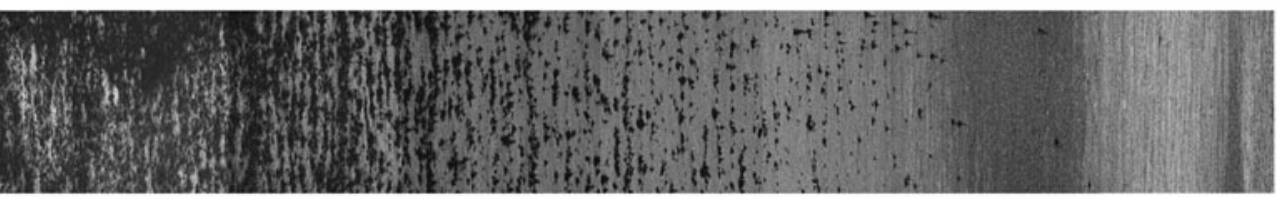

$\mathrm{Ti}-6-4(50 \%)+\mathrm{TiC}(50 \%)$ gradient

Ti-6-4

FIG. 7. Prototypes of gradient alloys fabricated using LD. (a-e) A Ti-6-4 to Nb rocket nozzle where the nozzle if fabricated from a high-temperature refractory metal, $\mathrm{Nb}$, and the body of the rocket is fabricated from low-density Ti-6-4. (f-j) The fabrication of $304 \mathrm{~L}$ to Inconel 626 valve stems where the valve is high-temperature Inconel 625 and the stem is $304 \mathrm{~L}$ stainless steel. This part is typically made using a friction weld. (k-n) A $304 \mathrm{~L}$ to Invar 36 gradient mirror with isogrid backing for increased stiffness. (o) A 304L to Invar 36 gradient alloy without any surface machining, showing the difference in oxide between the two alloys. (p) The alloy from (o) after surface machining and laser welding of a 304L plate, demonstrating weldability of the gradient alloy. (q) A successful gradient from Ti-6-4 to V, then from $\mathrm{V}$ to 420 stainless steel. (r) A similar gradient alloy to (q) but with a transition from Ti-6-4 to V, then from V to 304L, showing a crack. (s) After polishing, the crack at a specific composition is visible. (t) SEM micrograph showing that a brittle Fe-V-Cr phase has resulted in the cracking of the gradient alloy. (u) At Ti-6-4 to TiC gradient alloy. (v) Tiled micrographs from the gradient in (u) where the black phase is the TiC particles. This demonstrates the formation of a metal matrix composite using LD.

be increased to change the volume fraction of the inclusion. Several Ti-TiC gradient alloys were produced where the volume fraction of $\mathrm{TiC}$ was increased until cracking occurred. The gradient alloys shown in Figs. 7(u) and 7(v) are pure $\mathrm{Ti}$ on one side and are $50 \%$ by volume $\mathrm{TiC}$ on the other, which was the largest attainable volume fraction. Optical micrographs from the alloy are shown in Fig. 7(v) to demonstrate the gradient visually. 
TABLE I. Key mechanical and physical properties of the alloys used in the current work as measured on wrought material (not produced through AM). The properties shown are yield strength, density, Vicker's hardness, CTE from 293 to $473 \mathrm{~K}$, and the solidus temperature (which is the melting temperature for pure metals). All the data were taken from www.matweb.com. Compositionally graded alloys allow each side of the part to exhibit the properties of base material used, which allows for gradient of properties, such as density or CTE.

\begin{tabular}{|c|c|c|c|c|c|}
\hline Alloy & Yield strength (MPa) & Density $\left(\mathrm{g} \mathrm{cm}^{-3}\right)$ & Hardness (Vickers) & CTE $\mu \mathrm{m} \mathrm{m}^{-1} \mathrm{~K}^{-1}$ & Solidus temperature $(\mathrm{K})$ \\
\hline Ti-6Al-4V (annealed) & 880 & 4.4 & 349 & 8.6 & 1877 \\
\hline Titanium (annealed) & 140 & 4.5 & 60 & 8.9 & 1923 \\
\hline $\mathrm{TiC}$ & 258 & 4.9 & 3200 & 7.7 & 3323 \\
\hline Vanadium (annealed) & 454 & 6.1 & 159 & 8.3 & 2008 \\
\hline Niobium (annealed) & 207 & 8.6 & 80 & 7.1 & 2741 \\
\hline 304L stainless steel & 210 & 8.0 & 159 & 17.3 & 1673 \\
\hline 316L stainless steel & 240 & 8.0 & 155 & 16 & 1643 \\
\hline Invar 36 & 276 & 8.0 & 130 & 1.3 & 1700 \\
\hline Inconel 625 & 460 & 8.4 & 213 & 12.8 & 1563 \\
\hline
\end{tabular}

Table I lists some of the annealed literature values for the alloys which were compositionally graded in the current work along with selected mechanical property data to show the disparity in properties that the process allows. The five selected properties (yield strength, density, hardness, thermal expansion coefficient, and solidus temperature) for these alloys demonstrate a wide variable space for creating multifunctional parts. Of course, these values represent measurements performed on wrought material and ignore the many compositional variants that would exist in a gradient alloy between these. As such, future work should be directed at how mechanical and thermophysical properties vary across gradient alloys and how the ends of the 3D printed parts compare with standard wrought material.

\section{FUTURE DIRECTIONS FOR COMPOSITIONALLY GRADED METALS}

The current work demonstrates that advanced 3D printing techniques, which are being widely exploited in monolithic metals and polymers, can be extended to create multifunctional alloys with properties that cannot be obtained using other techniques. The future uses of the technology are broad, including such applications as predictive phase diagram modeling, new gradient compositions, exploitation of mechanical properties, mesoscale modeling of functionality, and the development of composite structures. From a manufacturing perspective, new processes will be developed to best exploit compositionally graded alloys to produce specialty hardware or for mass production.

As the gradient metallurgy field evolves, it is likely that technology will be adapted to accommodate the process. For example, a multihead LD system that is fed by the same powder source and moved by the same build head but that is capable of fabricating an array of gradient parts simultaneously could be envisioned. This technology aims to solve one of the largest detractors of adopting AM technology; the slow build times associated with one build-head rastering back and forth repeatedly to build a part. Using AM technology for mass production will require a rethinking of manufacturing away from the prototyping machines that exist today and into robust commercially viable machines.

\section{SUMMARY}

To conclude, the gradient LD process fundamentally changes the way that metal alloys can be fabricated and properties tuned. Unlike conventional metallurgical techniques of surface treating, heat treating, or creating metal matrix composites, for example, compositionally graded metallurgy allows for the strategic blending of materials in such a way that there is greater access to material property space than is traditionally available. The combination of blended metals in a single part with the freeform fabrication possibilities of three-dimensional printing makes the process unlike any conventional metallurgy technique. In the current work, it has been demonstrated that there is a clear roadmap for developing gradient alloys with advantageous mechanical properties. First, a desired application drives the gradient selection and design, including the required mechanical or physical properties (e.g., thermal expansion, density, melting temperature, hardness, toughness, among others). Second, phase diagrams are used to develop a compositional map which avoids brittle phases. Lastly, the geometry and architecture of the gradient are designed to be compatible with the LD process. In combination, this roadmap allows materials and applications to be developed which cannot be obtained using other techniques.

\section{ACKNOWLEDGMENTS}

This research was carried out at the Jet Propulsion Laboratory, California Institute of Technology, under a contract with the National Aeronautics and Space Administration (NASA) and funded through the Office of the Chief Technologist. The authors acknowledge A. Eilenberg, G. Agnes, A. Shapiro, C. Bradford, 
P. Gardner, C. Morandi, J. Mulder, P. Willis, and RPM Innovations for useful discussions. Richard Otis and Zi-Kui Liu acknowledge partial funding of this work by the Open Manufacturing Program of the Defense Advanced Research Projects Agency's Center for Innovative Materials Processing through Direct Digital Deposition at Penn State under Grant N00014-12-1-0840 from the Office of Naval Research.

\section{REFERENCES}

1. N. Hopkinson, R. Hague, and P. Dickens: Rapid Manufacturing: An Industrial Revolution for a Digital Age (Wiley-Blackwell, Berlin, Germany, 2005).

2. R.I. Campbell, R.J.M. Hague, B. Sener, and P.W. Wormald: The potential for the bespoke industrial designer. Des. J. 6, 24-34 (2003).

3. R.J.M. Hague, R.I. Campbell, and P.M. Dickens: Implications on design of rapid manufacturing. Proc. Inst. Mech. Eng., Part C 217, 25-30 (2003).

4. I. Gibson, D.W. Rosen, and B. Stucker: Additive Manufacturing Technologies: Rapid Prototyping to Direct Digital Manufacturing (Springer, New York, 2010).

5. E.C. Santos, M. Shiomi, K. Osakada, and T. Laoui: Rapid manufacturing of metal components by laser forming. Int. J. Mach. Tools Manuf. 46, 1459-1468 (2006).

6. M.L. Griffith, M.T. Ensz, J.D. Puskar, C.V. Robino, J.A. Brooks, J.A. Philliber, J.E. Smugeresky, and W.H. Hofmeister: Understanding the microstructure and properties of components fabricated by laser engineered net shaping (LENS). MRS Proc. 625, 9 (2011).

7. A. Crespo and R. Vilar: Finite element analysis of the rapid manufacturing of $\mathrm{Ti}-6 \mathrm{Al}-4 \mathrm{~V}$ parts by laser powder deposition. Scr. Mater. 63, 140-143 (2010).

8. S. Kelly and S. Kampe: Microstructural evolution in laser-deposited multilayer Ti-6Al-4V builds: Part I. Microstructural characterization Metall. Mater. Trans. 35, 1861-1867 (2004).

9. L.E. Murr, S.A. Quinones, S.M. Gaytan, M.I. Lopez, A. Rodela, E.Y. Martinez, D.H. Hernandez, E. Martinez, F. Medina, and R.B. Wicker: Microstructure and mechanical behavior of Ti-6Al-4V produced by rapid-layer manufacturing, for biomedical applications. J. Mech. Behav. Biomed. Mater. 2, 20-32 (2009).

10. H. Tan, F. Zhang, J. Chen, X. Lin, and W. Huang: Microstructure evolution of laser solid forming of Ti-Al-V ternary system alloys from blended elemental powders. Chin. Opt. Lett. 9, 051403-051406 (2011).
11. K.I. Schwendner, R. Banerjee, P.C. Collins, C.A. Brice, and H.L. Fraser: Direct laser deposition of alloys from elemental powder blends. Scr. Mater. 45, 1123-1129 (2001).

12. L. Xue and M.U. Islam: Free-form laser consolidation for producing metallurgically sound and functional components. J. Laser Appl. 12, 160 (2000).

13. R. Banerjee, P.C. Collins, D. Bhattacharyya, S. Banerjee, and H.L. Fraser: Microstructural evolution in laser deposited compositionally graded $\alpha / \beta$ titanium-vanadium alloys. Acta Mater. 51, 3277-3292 (2003).

14. P.C. Collins, R. Banerjee, S. Banerjee, and H.L. Fraser: Laser deposition of compositionally graded titanium-vanadium and titanium-molybdenum alloys. Mater. Sci. Eng., A 352, 118-128 (2003).

15. M.B. Bever and P.F. Duwez: Gradients in composite materials. Mater. Sci. Eng. 10, 1-8 (1972).

16. M. Shen and M.B. Bever: Gradients in polymeric materials. J. Mater. Sci. 7, 741-746 (1972).

17. K.M.B. Taminger and R.A. Hafley: Electron beam freeform fabrication: A rapid metal deposition process. In Proc. of the 3rd Ann. Auto. Comp. Conf., 2003; pp. 1-6.

18. J.K. Watson, K.M.B. Taminger, R.A. Hafley, and D.D. Petersen: Development of a prototype electron beam freeform fabrication system. In Proc. of 13th SFF Symp., 2002; pp. 458-465.

19. C.A. Brice and D.S. Henn: Rapid prototyping and freeform fabrication via electron beam welding deposition. Proceeding of International Institute of Welding Conference, Copenhagen, Denmark (2002).

20. D.C. Hofmann, J-Y. Suh, A. Wiest, G. Duan, M-L. Lind, M.D. Demetriou, and W.L. Johnson: Designing metallic glass matrix composites with high toughness and tensile ductility. Nature, 451 (2008), 1085-1089.

21. D.C. Hofmann, J-Y. Suh, A. Wiest, M-L. Lind, M.D. Demetriou, and W.L. Johnson: Development of tough, low-density titaniumbased bulk metallic glass matrix composites with tensile ductility. Proc. Natl. Acad. Sci. U. S. A. 105, 20136-20140 (2008).

22. Z-K. Liu: First-principles calculations, and CALPHAD modeling of thermodynamics. J. Phase Equilib. Diffus. 30, 517-534 (2009).

23. J-O. Andersson, T. Helander, L. Höglund, P. Shi, and B. Sundman: Thermo-Calc \& DICTRA, computational tools for materials science. CALPHAD 26, 273-312 (2002).

24. L. Kaufman and H. Bernstein: Computer Calculation of Phase Diagrams with Special Reference to Refractory Metal (Academic Press, New York, NY, 1970).

25. See for example, http://www.nasa.gov/exploration/systems/sls/ 3d-printed-rocket-injector.html\#.U5IRMU1OVaQ, NASA Test Limits of 3-D Printing with Powerful Rocket Engine Check, 2013. 